OPEN ACCESS

Edited by:

Mahendra Pratap Kashyap,

University of Alabama at Birmingham,

United States

Reviewed by:

Siva Koganti,

Stony Brook Medicine, United States

Jun Cheng,

Shenzhen University, China

*Correspondence:

Lu Zheng

xqyyz11@163.com

${ }^{\dagger}$ Qinqin Liu and Nan You contributed equally to this study.

Specialty section:

This article was submitted to

Molecular Diagnostics and

Therapeutics,

a section of the journal

Frontiers in Molecular Biosciences

Received: 10 June 2021 Accepted: 28 September 2021 Published: 26 November 2021

Citation:

Liu Q, You N, Zhu J, Li J, Wu K, Wang Z, Wang L, Zhu Y, Gu H, Peng $X$ and Zheng $L$ (2021) A Nomogram Model for Predicting the Response to Transcatheter Arterial Embolization in Patients With Symptomatic Hepatic Hemangioma.

Front. Mol. Biosci. 8:722864. doi: 10.3389/fmolb.2021.722864

\section{A Nomogram Model for Predicting the Response to Transcatheter Arterial Embolization in Patients With Symptomatic Hepatic Hemangioma}

\author{
Qinqin Liu ${ }^{1,2 \dagger}$, Nan You ${ }^{1 \dagger}$, Jiangqin Zhu ${ }^{1}$, Jing $\mathrm{Li}^{1}, \mathrm{Ke} \mathrm{Wu}^{1}$, Zheng Wang ${ }^{1}$, Liang Wang ${ }^{1}$, \\ Yinan Zhu ${ }^{1}$, Huiying $\mathrm{Gu}^{1}$, Xuehui Peng ${ }^{1}$ and Lu Zheng ${ }^{1 *}$ \\ ${ }^{1}$ Department of Hepatobiliary Surgery, the Second Affiliated Hospital of Army Medical University, Chongqing, China, ${ }^{2}$ Department \\ of Biliary-Pancreatic Surgery, Sun Yat-Sen Memorial Hospital, Sun Yat-Sen University, Guangzhou, China
}

Background: Transcatheter arterial embolization (TAE) is regarded as an effective treatment for patients with symptomatic hepatic hemangioma. However, few studies have evaluated the efficacy of TAE alone for treating hepatic hemangioma. The aim of this study was to identify the factors that influence the response to TAE and formulate a quantitative nomogram to optimize the individualized management of hepatic hemangioma.

Methods: We retrospectively studied 276 patients treated with TAE for hepatic hemangioma at our center from January 2011 to December 2019. The full cohort was randomly divided into training and validation cohorts. After assessing the potential predictive factors for the efficacy of TAE in the training cohort, a nomogram model was established and evaluated by discrimination and calibration.

Results: During follow-up, the symptom relief rate was 100\%. The tumor blood supply $(p<0.001)$, tumor number $(p=0.004)$, and tumor size $(p=0.006)$ were identified as significant predictors of the failure of tumor shrinkage in response to TAE. The nomogram model showed favorable discrimination and calibration, with a C-index of 0.775 (95\% Cl, $0.705-0.845)$ in the training cohort, which was further confirmed in the validation cohort (C-index $0.768 ; 95 \% \mathrm{Cl}, 0.680-0.856$ ). The side effects of TAE were relatively minor and included mainly abdominal pain, nausea, vomiting, fever, and the presence of elevated hepatic transaminases.

Conclusion: TAE is a safe and effective treatment for symptomatic hepatic hemangioma. The established nomogram performed well for the estimation of the effect of TAE in patients with hepatic hemangioma and can facilitate the selection of patients who would benefit most from the treatment.

Keywords: nomogram, therapeutic response, transcatheter arterial embolization, symptomatic hepatic hemangioma, efficacy

Abbreviations: HBsAg, hepatitis B surface antigen; WBC, white blood cell; PLT, platelet; HGB, hemoglobin; TBil, total bilirubin; ALT, alanine aminotransferase; AST, aspartate transaminase; TAE, transcatheter arterial embolization. 


\section{INTRODUCTION}

Hepatic hemangioma is the most common benign vascular lesion of the liver with an approximate incidence ranging from 0.4 to 20\% (Choi and Nguyen, 2005; Fang et al., 2015). Hepatic hemangioma is congenital vascular malformations caused by vascular developmental abnormalities and abnormal differentiation, which expands during pregnancy and may be associated with the administration of estrogen (Chatzoulis et al., 2008; Duxbury and Garden, 2010; Ketchum et al., 2019). Most cases are found by chance with a high prevalence of accurate and sensitive imaging modalities. Small and asymptomatic lesions require no active treatment or monitoring (Herman et al., 2005; Schnelldorfer et al., 2010; Hasan et al., 2014). However, a small number of hepatic hemangiomas grow progressively and present as abdominal pain, distention, fatigue, anemia, obstructive jaundice, Kasabach-Merritt syndrome, and spontaneous rupture, which require clinical management (Donati et al., 2011; Toro et al., 2014; Liu et al., 2018).

The optimal selection of management strategies for expanding and symptomatic hepatic hemangioma remains controversial (Özden et al., 2017). Among them, transcatheter arterial embolization (TAE) is a promising, minimally invasive technique for the treatment of symptomatic hepatic hemangioma, associated with symptom relief and marked tumor shrinkage (Srivastava et al., 2001). A multicenter study involving 836 cases reported that transarterial chemoembolization was safe and effective for treating giant hepatic hemangioma, and the results showed a $100 \%$ symptom relief rate and clear tumor regression (Li et al., 2015). Several studies have investigated the effect of TAE on hepatic hemangioma, but most of them were limited by a small number of patients and short-term data (Akhlaghpoor et al., 2018; Torkian et al., 2021). Nevertheless, different treatment responses were not assessed in these previous studies and patients who would benefit from TAE were not well defined. In addition, one recent study attached the importance to preoperative TAE for treating ruptured hepatic hemangioma, which showed reduced blood loss in the subsequent surgical resection (Ramachandran et al., 2010). Despite the promising results in controlling hepatic hemangioma, some patients experienced failure to benefit from TAE. The present study aimed to investigate the potential factors correlating with the efficacy of TAE and to develop a nomogram for the management of individual hepatic hemangioma.

\section{MATERIALS AND METHODS}

\section{Patients}

A cohort of 276 patients diagnosed with hepatic hemangioma, who underwent TAE only from January 2011 to December 2019 in the Second Affiliated Hospital of Army Medical University, were retrospectively collected. The inclusion criteria were as follows: 1) hepatic hemangioma confirmed by contrastenhanced computed tomography (CT) or magnetic resonance imaging (MRI); 2) the presence of hepatic hemangioma-related symptoms and/or complications, such as abdominal complaints and intraabdominal hemorrhage; 3) Child-Pugh A liver function; 4) no other combined malignancies; 5) availability of complete clinicopathological and follow-up data; and 6) patients who did not receive other treatment options during the course of the disease. 276 eligible patients were ultimately enrolled and randomly divided into training $(60 \%, \mathrm{n}=166)$ and validation $(40 \%, n=110)$ cohorts. The study was performed with approval from the Ethics Committee of the Second Affiliated Hospital of Army Medical University, and all patients signed written informed consent forms. All authors had access to the study data and reviewed and approved the final manuscript.

\section{TAE Procedure and Follow-Up}

Under local anesthesia, all enrolled individuals underwent selective arteriography of their superior mesenteric, celiac, and common hepatic arteries to determine the location of the tumor and its blood supply. A mixture of pingyangmycin or bleomycin with lipiodol was slowly injected through a 5-Fr catheter into the tumor-feeding branches of the blood vessels. The amount of mixture injected depended on the size and number of the lesions. Embolization was conducted until complete tumor arterial flow stasis was observed on an angiogram.

The following variables were included for analysis: age, sex, oral contraceptives, hepatitis B surface antigen (HBsAg), comorbidities, tumor location, tumor distribution, tumor size, tumor number, tumor blood supply, white blood cell (WBC), platelet (PLT), hemoglobin (HGB), total bilirubin (TBil), alanine aminotransferase (ALT), aspartate transaminase (AST), drugs used for TAE, the number of TAEs, follow-up time, and postoperative complications. In addition, hemangioma-related symptom relief was assessed and recorded.

Every enrolled patient received the CT scan 1 month after the initiation of treatment and then every 3-6 months thereafter to evaluate the therapeutic response. The tumor response to TAE was based on the Response Evaluation Criteria in Solid Tumors (RECIST) (Duffaud and Therasse, 2000), and all patients were stratified into an effective group (complete response, $\mathrm{CR},+$ partial response, $\mathrm{PR}$ ) and an ineffective group (stable disease, $\mathrm{SD},+$ progressive disease, $\mathrm{PD}$ ). The TAE procedure was repeated according to the therapeutic efficacy and patient tolerance.

\section{Tumor Blood Supply}

The CT value was measured at the largest cross-section of the tumor in the arterial enhancement phase before TAE, and the average CT value of all the tumors (CT1) was obtained. To better evaluate the blood supply of the lesions, the liver parenchyma CT value (CT2) in the arterial enhancement phase was measured. Subsequently, tumor blood supply was categorized as hypovascular or hypervascular based on the median CT1/ CT2 value.

\section{Model Construction and Evaluation}

The patients were randomly assigned into the training and validation cohorts with a split ratio of $3: 2$. Univariate and multivariate analyses were performed on the training cohort to determine the significant independent factors for which a 
TABLE 1 | Demographic characteristics of patients with hepatic hemangioma in the training and validation cohorts.

\begin{tabular}{|c|c|c|c|c|c|c|}
\hline \multirow[t]{2}{*}{ Variables } & \multicolumn{2}{|c|}{ Training cohort $(n=166)$} & \multirow[t]{2}{*}{$P$} & \multicolumn{2}{|c|}{ Validation cohort $(n=110)$} & \multirow[t]{2}{*}{$P$} \\
\hline & Effective & Ineffective & & Effective & Ineffective & \\
\hline Age, years & $46.0(40.0-53.3)$ & $46.0(41.3-51.8)$ & 0.911 & $47.0(41.0-52.3)$ & $45.0(39.0-52.8)$ & 0.465 \\
\hline Sex & - & - & 0.490 & - & - & 0.123 \\
\hline Male & 29 (33.7\%) & 23 (28.8\%) & - & 23 (46.0\%) & 19 (31.7\%) & - \\
\hline Female & 57 (66.3\%) & $57(71.3 \%)$ & - & 27 (54.0\%) & $41(68.3 \%)$ & - \\
\hline Oral contraceptives & - & - & 0.245 & - & - & 0.310 \\
\hline Yes & 7 (8.1\%) & $11(13.8 \%)$ & - & 5 (10.0\%) & $10(16.7 \%)$ & - \\
\hline No & 79 (91.9\%) & 69 (86.3\%) & - & 45 (90.0\%) & 50 (83.3\%) & - \\
\hline $\mathrm{HBsAg}$ & - & - & 0.530 & - & - & 0.347 \\
\hline Positive & 12 (14.0\%) & $14(17.5 \%)$ & - & $8(16.0 \%)$ & $6(10.0 \%)$ & - \\
\hline Negative & 74 (86.0\%) & 66 (82.5\%) & - & 42 (84.0\%) & 54 (90.0\%) & - \\
\hline Comorbidities & - & - & 0.484 & - & - & 0.515 \\
\hline Present & 6 (7.0\%) & 8 (10.0\%) & - & 4 (8.0\%) & 2 (3.3\%) & - \\
\hline Absent & 80 (93.0\%) & 72 (90.0\%) & - & 46 (92.0\%) & 58 (96.7\%) & - \\
\hline Tumor location & - & - & 0.160 & - & - & 0.996 \\
\hline Left lobe & 49 (57.0\%) & 40 (50.0\%) & - & 26 (52.0\%) & 31 (51.7\%) & - \\
\hline Right lobe & 16 (18.6\%) & 10 (12.5\%) & - & $8(16.0 \%)$ & 10 (16.7\%) & - \\
\hline Bilobar & 21 (24.4\%) & 30 (37.5\%) & - & 16 (32.0\%) & 19 (31.7\%) & - \\
\hline Tumor distribution & - & - & 0.132 & - & - & 0.167 \\
\hline Subcapsular & 23 (26.7\%) & 22 (27.5\%) & - & 20 (40.0\%) & 14 (23.3\%) & - \\
\hline Deep situated & 56 (62.8\%) & 41 (51.3\%) & - & 24 (48.0\%) & 36 (60.0\%) & - \\
\hline Both & 9 (10.5\%) & 17 (21.3\%) & - & 6 (12.0\%) & 10 (16.7\%) & - \\
\hline Tumor size, cm & $6.1(4.8-7.2)$ & $6.7(5.5-8.1)$ & 0.020 & $6.0(4.8-7.5)$ & $6.9(5.3-8.0)$ & 0.023 \\
\hline Tumor number & - & - & $<0.001$ & - & - & 0.001 \\
\hline Solitary & 56 (65.1\%) & 30 (37.5\%) & - & 32 (64.0\%) & 20 (33.3\%) & - \\
\hline Multiple & 30 (34.9\%) & $50(62.5 \%)$ & - & 18 (36.0\%) & 40 (66.7\%) & - \\
\hline Tumor blood supply & - & - & $<0.001$ & - & - & 0.001 \\
\hline Hypovascular & 26 (30.2\%) & $51(63.8 \%)$ & - & 19 (38.0\%) & 42 (70.0\%) & - \\
\hline Hypervascular & 60 (69.8\%) & 29 (36.3\%) & - & 31 (62.0\%) & 18 (30.0\%) & - \\
\hline WBC, $10^{9} / \mathrm{L}$ & $5.2(4.5-5.9)$ & $5.0(4.3-6.0)$ & 0.525 & $5.5(4.5-6.3)$ & $5.0(4.4-6.1)$ & 0.515 \\
\hline $\mathrm{PLT}, 10^{9} / \mathrm{L}$ & $188.0(138.8-230.0)$ & 174.5 (146.3-213.3) & 0.439 & $175.0(141.0-208.8)$ & 198.5 (162.0-221.3) & 0.089 \\
\hline $\mathrm{HGB}, \mathrm{g} / \mathrm{L}$ & $132.0(121.8-142.0)$ & $127.5(118.0-140.0)$ & 0.189 & $132.5(119.8-143.0)$ & $127.0(114.3-138.0)$ & 0.107 \\
\hline TBIL, umol/L & $11.9(9.1-15.6)$ & $13.1(9.3-17.3)$ & 0.616 & $12.0(9.2-15.7)$ & $12.1(9.9-17.6)$ & 0.307 \\
\hline $\mathrm{ALT}, \mathrm{IU} / \mathrm{L}$ & 19.0 (13.8-28.0) & $16.0(12.1-25.2)$ & 0.121 & 19.0 (13.0-27.5) & $16.0(12.2-23.4)$ & 0.174 \\
\hline AST, IU/L & 18.0 (16.0-24.3) & 18.1 (15.2-23.0) & 0.634 & 19.5 (15.8-24.0) & 18.0 (15.9-22.5) & 0.644 \\
\hline Drug & - & - & 0.617 & - & - & 0.152 \\
\hline Pinyangmycin & 58 (67.4\%) & 51 (63.8\%) & - & 38 (76.0\%) & 38 (63.3\%) & - \\
\hline Bleomycin & 28 (32.6\%) & 29 (36.3\%) & - & 12 (24.0\%) & 22 (36.7\%) & - \\
\hline Repeated TAE & - & - & 0.030 & - & - & 1.000 \\
\hline 0 & 85 (98.8\%) & 72 (90.0\%) & - & 49 (98.0\%) & 58 (96.7\%) & - \\
\hline 1 & 1 (1.2\%) & 8 (10.0\%) & - & 1 (2.0\%) & 2 (3.3\%) & - \\
\hline
\end{tabular}

predictive nomogram was indicated. The discrimination ability of the nomogram was determined using the concordance index (C-index). The calibration curve for predictive accuracy of the nomogram was used to analyze the consistency between the predicted and observed probability.

\section{Statistical Analysis}

Statistical analyses were performed using SPSS version 22.0 (IBM SPSS, Inc., Chicago, IL) and R version 3.5.1 (http://www.rproject.org). The continuous variables were summarized using the median with the interquartile range and compared using the $t$-test or Mann-Whitney-Wilcoxon test, while the categorical variables were expressed as frequency with proportion and compared using the chi-square test or Fisher's exact test. Univariate and multivariate logistic regression analyses were employed to identify the significantly associated factors. Variables with $p<0.05$ in the univariate analysis were entered into the multivariate analysis. A nomogram was constructed based on the independent predictors identified above using the rms $\mathrm{R}$ package. The discrimination and calibration of the nomogram were assessed using the $\mathrm{C}$-index and the calibration curve. $p$ values $<0.05$ were considered statistically significant.

\section{RESULTS}

\section{Baseline Characteristics}

In total, 276 patients who received TAE for hepatic hemangioma were enrolled in this study. Baseline characteristics of the training and validation cohorts are presented in Table 1. No statistically significant differences were observed between the two cohorts regarding tumor response to TAE, with response rates of 51.8 and $45.5 \%$ in the training and validation cohorts, respectively ( $p=$ 
TABLE 2 | Univariate and multivariate analyses of predictive factors associated with tumor response to TAE in the training cohort.

\begin{tabular}{|c|c|c|c|c|}
\hline \multirow[t]{2}{*}{ Variables } & \multirow{2}{*}{$\frac{\text { Univariate analysis }}{\text { Hazard ratio }(95 \% \mathrm{Cl})}$} & \multirow[t]{2}{*}{$p$} & \multirow{2}{*}{$\frac{\text { Multivariate analysis }}{\text { Hazard ratio }(95 \% \mathrm{Cl})}$} & \multirow[t]{2}{*}{$p$} \\
\hline & & & & \\
\hline Age, years & 0.997 (0.964-1.031) & 0.853 & - & - \\
\hline Sex & - & - & - & - \\
\hline Male & Reference & - & - & - \\
\hline Female & $1.261(0.652-2.437)$ & 0.491 & - & - \\
\hline Oral contraceptives & & - & - & - \\
\hline No & Reference & - & - & - \\
\hline Yes & $1.799(0.661-4.896)$ & 0.250 & - & - \\
\hline $\mathrm{HBsAg}$ & - & - & - & - \\
\hline Negative & Reference & - & - & - \\
\hline Positive & 1.308 (0.565-3.028) & 0.531 & - & - \\
\hline Comorbidities & - & - & - & - \\
\hline Absent & Reference & - & - & - \\
\hline Present & $1.481(0.491-4.474)$ & 0.486 & & - \\
\hline Tumor location & - & - & - & - \\
\hline Left lobe & Reference & - & - & - \\
\hline Right lobe & $0.766(0.313-1.871)$ & 0.558 & - & - \\
\hline Bilobar & $1.750(0.872-3.513)$ & 0.115 & - & - \\
\hline Tumor distribution & & - & - & - \\
\hline Subcapsular & Reference & - & - & - \\
\hline Deep situated & $0.794(0.390-1.617)$ & 0.525 & - & - \\
\hline Both & $1.975(0.728-5.353)$ & 0.181 & - & - \\
\hline Tumor size, cm & $1,229(1.051-1.437)$ & 0.010 & $1.293(1.075-1.554)$ & 0.006 \\
\hline Tumor number & - & - & - & - \\
\hline Solitary & Reference & - & Reference & - \\
\hline Multiple & $3.111(1.651-5.863)$ & $<0.001$ & $2.825(1.402-5.695)$ & 0.004 \\
\hline Tumor blood supply & & - & - & - \\
\hline Hypervascular & Reference & - & Reference & - \\
\hline Hypovascular & $4.058(2.123-7.756)$ & $<0.001$ & $5.150(2.468-10.743)$ & $<0.001$ \\
\hline WBC, $10^{9} / \mathrm{L}$ & $0.869(0.707-1.068)$ & 0.182 & - & - \\
\hline PLT, $10^{9} / \mathrm{L}$ & 0.998 (0.993-1.003) & 0.386 & - & - \\
\hline $\mathrm{HGB}, \mathrm{g} / \mathrm{L}$ & $0.990(0.971-1.009)$ & 0.287 & - & - \\
\hline TBIL,umol/L & $1.024(0.970-1.078)$ & 0.392 & - & - \\
\hline ALT,IU/L & $0.992(0.976-1.008)$ & 0.310 & - & - \\
\hline AST,IU/L & $0.995(0.966-1.024)$ & 0.714 & - & - \\
\hline Drug & - & - & - & - \\
\hline Pinyangmycin & Reference & - & - & - \\
\hline Bleomycin & $1.178(0.620-2.237)$ & 0.617 & - & - \\
\hline Repeated TAE & - & - & - & - \\
\hline 0 & Reference & - & Reference & - \\
\hline 1 & $9.444(1.154-77.312)$ & 0.036 & $7.670(0.756-77.850)$ & 0.085 \\
\hline
\end{tabular}

0.301). Tumor size, tumor number, and tumor blood supply differed significantly between the two groups in both the training and validation cohorts, and a larger number of patients were repeatedly treated with TAE in the ineffective group of the training cohort. The median follow-up duration was 3.4 $(2.0-5.3)$ years for patients in the training cohort and 3.3 $(1.4-5.3)$ years for patients in the validation cohort $(p=0.832)$.

\section{Complication and Symptomatic Improvement}

The side effects of embolization were assessed in the present study, including abdominal pain in 167 cases (60.5\%), nausea or vomiting in 124 cases (44.9\%), fever in 73 cases $(26.4 \%)$, and elevation of hepatic transaminases in 31 cases $(11.2 \%)$. These symptoms were limited to 3-4 days and required no treatment or were easily controlled with symptomatic treatment. No serious complications were observed. All patients showed reduced symptoms from the intervention of TAE.

\section{Univariate and Multivariate Analyses of the Predictive Factors Associated With Tumor Response to TAE in Patients With Hepatic Hemangioma}

The univariate and multivariate analyses of the training cohort were employed to investigate the predictors of the response to TAE in patients with hepatic hemangioma (Table 2). By assessing multiple potential predictive factors, the variables of tumor size, tumor number, tumor blood supply, and repeated TAE were determined to be related to the efficacy of TAE. Furthermore, the results of the multivariate analysis showed that tumor blood supply $(\mathrm{HR}=5.150 ; 95 \% \mathrm{CI}=2.468-10.743 ; p<0.001)$, tumor number $(\mathrm{HR}=2.825 ; 95 \% \mathrm{CI}=1.402-5.695 ; p=0.004)$, and 


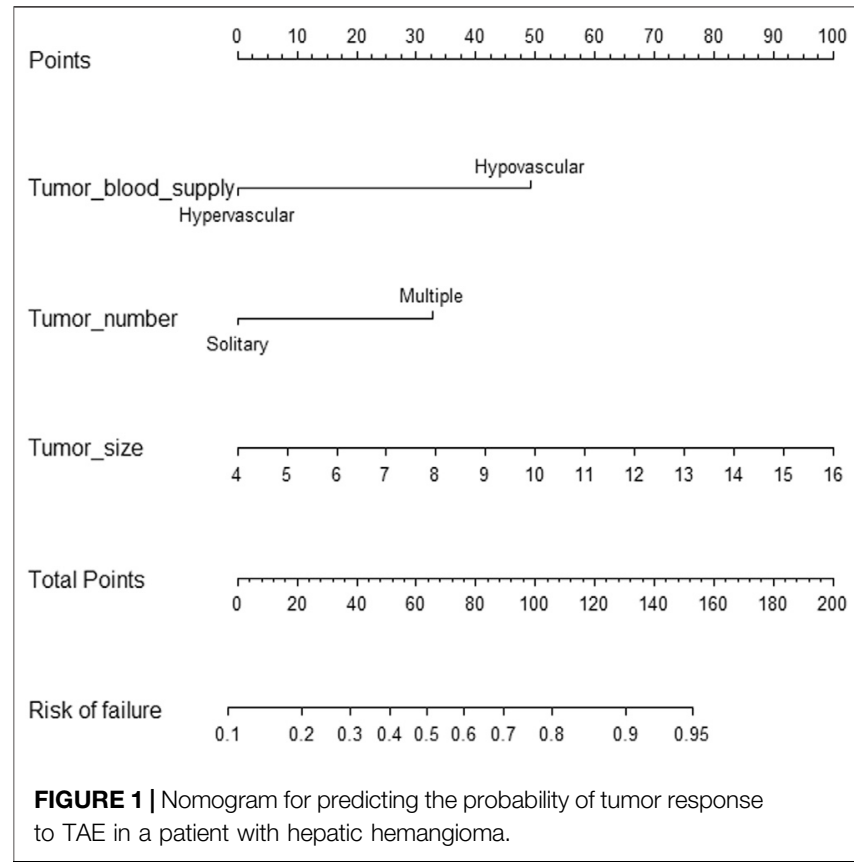

tumor size $(\mathrm{HR}=1.293 ; 95 \% \mathrm{CI}=1.075-1.554 ; p=0.006)$ were significant predictors of the failure of tumor shrinkage in response to TAE.

\section{Nomogram Construction and Validation}

The nomogram incorporating the independent variables, namely, tumor size, tumor number, and tumor blood supply, identified above, was constructed to estimate patients' personalized therapeutic response to TAE (Figure 1). The nomogram yielded a C-index of 0.775 (95\% CI, 0.705-0.845) for the training cohort and a C-index of 0.768 ( $95 \%$ CI, $0.680-0.856)$ for the validation cohort. Based on the projecting total score onto the lower total point scale by adding the scores of each variable, the probability of tumor response to TAE in a patient with hepatic hemangioma can be estimated. The calibration curve indicated the optimal agreement between nomogram-predicted probability and the result of observation in both the training and validation cohorts (Figure 2).

\section{DISCUSSION}

Symptomatic hepatic hemangioma is a common hepatic neoplasm that requires effective intervention. Among various treatment options for patients with hepatic hemangioma, TAE is accepted as a less invasive method associated with fewer complications and quicker recovery (Szejnfeld et al., 2015). However, not all patients respond well to TAE, evidenced by the heterogeneous tumor response. In this study, we analyzed the clinical data of 276 patients who received TAE for symptomatic hepatic hemangioma, and an efficacy estimation nomogram was created. To the best of our knowledge, the study is the first exploration of the potential therapeutic effect on TAE in patients with hepatic hemangioma.

Surgical resection is technically difficult and associated with severe complications and a long recovery time (Yoon et al., 2003; Miura et al., 2014). Although microwave ablation and radiofrequency ablation are thought to be less risky treatments, the long ablation time and dangerous hemolysis may reduce the intervention benefits for hepatic hemangioma (Gao et al., 2013; Ji et al., 2016). In comparison, TAE is more applicable, and clinical remission and tumor size reduction can be observed. In addition, TAE can be used to downsize a giant hepatic hemangioma, thus facilitating the subsequent resection or ablation, thereby minimizing possible complications (Firouznia et al., 2014).
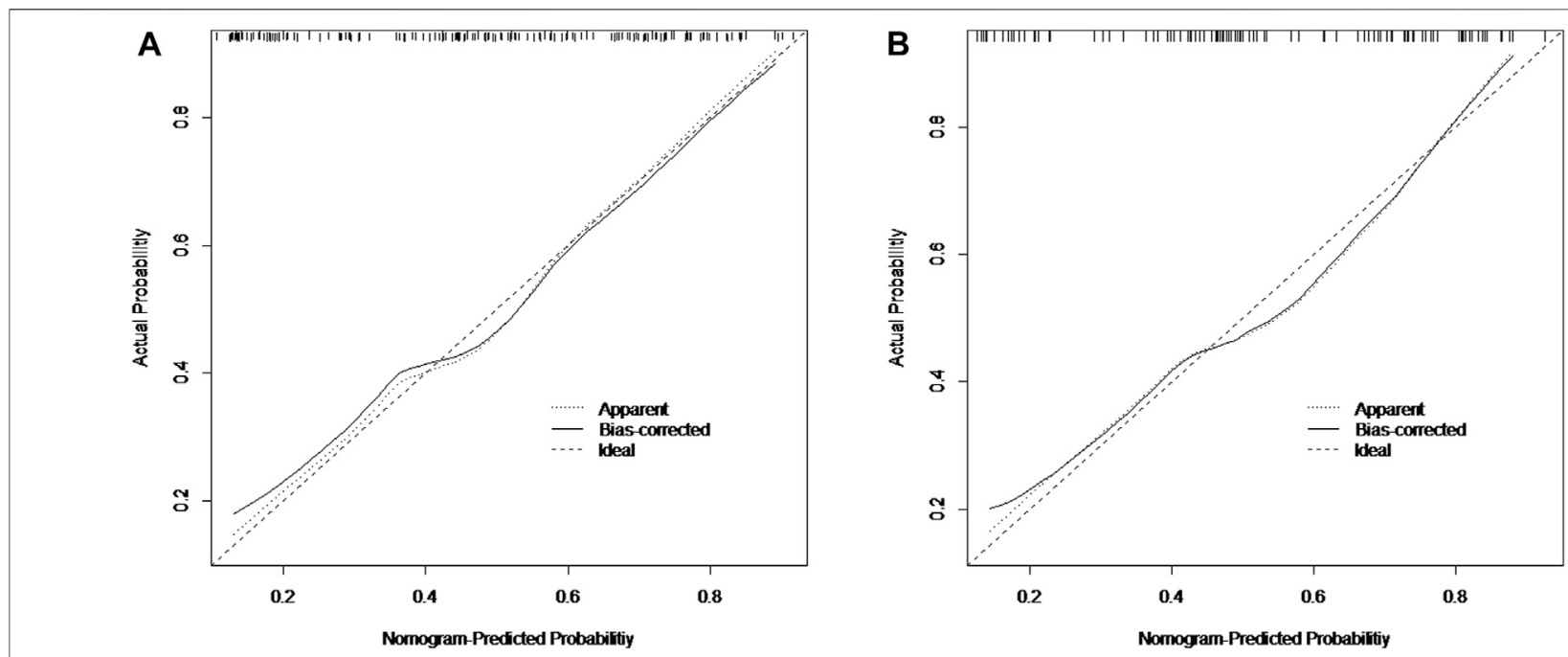

FIGURE 2 | Calibration curves of the nomogram for the training cohort (A) and the validation cohort (B). 
Our analysis demonstrated that there was improvement in the symptoms of all patients with symptomatic hepatic hemangioma treated with TAE, which is consistent with the findings of previous studies (Deutsch et al., 2001; Srivastava et al., 2001). Given that the shrinkage of hepatic hemangioma in response to TAE differed among patients, the potential predictive variables were further analyzed. This involved determining the significant pretreatment parameters that could be used for tumor response prediction. The nomogram was developed further to predict each patient's likelihood of response to TAE based on the factors, which showed good predictive performance with a $\mathrm{C}$-index of 0.775 (95\% CI, 0.705-0.845) for the training cohort, which was validated by the internal validation cohort with a C-index of 0.768 (95\% CI, 0.680-0.856). Thus, optimal candidates for TAE could be identified based on the individual probability of response to TAE. For lesions refractory to TAE, other suitable alternatives such as surgical resection should be considered.

In our nomogram model, tumor blood supply is identified as the greatest contributor to tumor response from TAE. The hypervascular lesion is significantly associated with increased efficacy of TAE. Previous studies indicated that hepatic hemangioma consists of blood-filled sinuses nourished by hepatic arteries (Giavroglou et al., 2003; Li et al., 2003). Superselective embolization of the multiple feeding vessels of the hepatic hemangioma allowed pinyangmycin or bleomycin to damage tumor-associated endothelial cells continuously and accelerate the progression of the occlusion and fibrosis of blood sinuses, thereby causing tumor regression (Szejnfeld et al., 2015). Thus, an improved accumulation of lipiodol is more likely to be achieved in a hypervascular lesion, resulting in increased probability of a positive therapeutic effect. Meanwhile, it is clear that hypovascular lesions are less sensitive to TAE because an insufficient quantity of embolic agents is deposited in the tumor.

Besides, tumor size and tumor number were associated with an increased risk of TAE treatment failure. Generally, TAE provides a hypoxic and anoxic environment to shrink the lesions by blocking the arterial blood supply (Xue et al., 2017). However, multifocal or giant hepatic hemangioma may recruit new blood vessels by angiogenesis and vasculogenesis, which reduces the long-term therapeutic efficacy in spite of repeated TAE. These cases with multiple feeding vessels, in which the effective dose of lipiodol is compromised by the tumor burden, are possible explanations for treatment failure. Although neovascularization may affect the tumor response, tumor shrinkage is progressive over time, and a satisfactory TAE response can be achieved in a single small lesion (Bozkaya et al., 2014). Consequently, other effective interventions or combined therapies are encouraged for patients with multinodular and large hepatic hemangioma.

The commonly reported side effects associated with TAE are abdominal pain, fever, nausea, and vomiting, which are caused by thrombosis and necrosis (Giavroglou et al., 2003). These complications were transient, most of which were resolved easily with conservative therapy ( $\mathrm{Li}$ et al., 2015). Serious side effects rarely occur, such as liver failure, hepatic abscess formation, and cholecystitis. Given the benign nature of hemangioma, TAE is the preferred treatment, due to the good efficacy and its association with minor side effects when compared with the surgical approach (Deutsch et al., 2001). In agreement with our initial favorable experience with TAE, no major TAE-related complications occurred, and only mild and transient symptoms were noted.

Although the nomogram showed favorable predictive accuracy for the probability of poor tumor response to TAE in patients with hepatic hemangioma, there are still some limitations that should be acknowledged. First, this is a retrospective study from a single institution, which may introduce some potential bias. Second, the small sample size in the established nomogram may affect the accurate assessment of treatment failure. Third, there was no external validation, and further evaluation is required before applying the model in clinical practice. Therefore, a further prospective study with a larger sample size is warranted to confirm the validity of the model.

In conclusion, TAE is a safe and effective treatment option with satisfactory symptom control and marked tumor shrinkage in appropriately selected patients. A novel nomogram integrating independent predictive variables was developed to predict the efficacy of TAE in patients with hepatic hemangioma, which demonstrated good accuracy and calibration and could assist clinicians to develop an individualized treatment strategy.

\section{DATA AVAILABILITY STATEMENT}

The original contributions presented in the study are included in the article, further inquiries can be directed to the corresponding author.

\section{ETHICS STATEMENT}

Written informed consent was obtained from the individual(s) for the publication of any potentially identifiable images or data included in this article.

\section{AUTHOR CONTRIBUTIONS}

NY, JL, and LZ contributed to the study conception and design. QL, KW, ZW, LW, YZ, XP, and HG collected the data. QL analyzed the data. QL drafted the manuscript, and the other authors revised the manuscript. All authors reviewed and approved the manuscript.

\section{FUNDING}

This study was supported by grants from the Medical Research Project jointly funded by the Chongqing Science and Technology Commission and the Chongqing Health Commission (2019ZDXM046), the Technological Innovation and Application Demonstration Special Project of Chongqing (cstc2018jscx-mszdX0012), and the Scientific and Technological Innovation Special Project of Army Medical University (2019XLC 2006). 


\section{REFERENCES}

Akhlaghpoor, S., Torkian, P., and Golzarian, J. (2018). Transarterial BleomycinLipiodol Embolization (B/LE) for Symptomatic Giant Hepatic Hemangioma. Cardiovasc. Intervent Radiol. 41, 1674-1682. doi:10.1007/s00270-018-2010-4

Bozkaya, H., Cinar, C., Besir, F. H., Parıldar, M., and Oran, I. (2014). Minimally Invasive Treatment of Giant Haemangiomas of the Liver: Embolisation with Bleomycin. Cardiovasc. Intervent Radiol. 37, 101-107. doi:10.1007/s00270-0130618-y

Chatzoulis, G., Kaltsas, A., Daliakopoulos, S., Sallam, O., Maria, K., Chatzoulis, K., et al. (2008). Co-existence of a Giant Splenic Hemangioma and Multiple Hepatic Hemangiomas and the Potential Association with the Use of Oral Contraceptives: a Case Report. J. Med. Case Rep. 2, 147. doi:10.1186/1752-19472-147

Choi, B. Y., and Nguyen, M. H. (2005). The Diagnosis and Management of Benign Hepatic Tumors. J. Clin. Gastroenterol. 39, 401-412. doi:10.1097/ 01.mcg.0000159226.63037.a2

Deutsch, G. S., Yeh, K. A., Bates, W. B., 3rd, and Tannehill, W. B. (2001). Embolization for Management of Hepatic Hemangiomas. Am. Surg. 67, 159-164.

Donati, M., Stavrou, G. A., Donati, A., and Oldhafer, K. J. (2011). The Risk of Spontaneous Rupture of Liver Hemangiomas: a Critical Review of the Literature. J. Hepato-Biliary-Pancreatic Sci. 18, 797-805. doi:10.1007/s00534011-0420-7

Duffaud, F., and Therasse, P. (2000). New Guidelines to Evaluate the Response to Treatment in Solid Tumors. Bull. Cancer 87, 881-886.

Duxbury, M. S., and Garden, O. J. (2010). Giant Haemangioma of the Liver: Observation or Resection. Dig. Surg. 27, 7-11. doi:10.1159/000268108

Fang, L., Zhu, Z., Huang, B., Ding, H., Mao, F., Li, C., et al. (2015). A Comparative Study of Contrast Enhanced Ultrasound and Contrast Enhanced Magnetic Resonance Imaging for the Detection and Characterization of Hepatic Hemangiomas. Bst 9, 104-110. doi:10.5582/bst.2015.01026

Firouznia, K., Ghanaati, H., Alavian, S. M., Nassiri Toosi, M., Ebrahimi Daryani, N., Jalali, A. H., et al. (2014). Management of Liver Hemangioma Using Transcatheter Arterial Embolization. Hepat. Mon 14, e25788. doi:10.5812/ hepatmon. 25788

Gao, J., Ke, S., Ding, X.-m., Zhou, Y.-m., Qian, X.-j., and Sun, W.-b. (2013). Radiofrequency Ablation for Large Hepatic Hemangiomas: Initial Experience and Lessons. Surgery 153, 78-85. doi:10.1016/j.surg.2012.06.004

Giavroglou, C., Economou, H., and Ioannidis, I. (2003). Arterial Embolization of Giant Hepatic Hemangiomas. CardioVascular Interv. Radiol. 26, 92-96. doi:10.1007/s00270-002-2648-8

Hasan, H. Y., Hinshaw, J. L., Borman, E. J., Gegios, A., Leverson, G., and Winslow, E. R. (2014). Assessing Normal Growth of Hepatic Hemangiomas during LongTerm Follow-Up. JAMA Surg. 149, 1266-1271. doi:10.1001/jamasurg.2014.477

Herman, P., Costa, M., Machado, M., Pugliese, V., Dalbuquerque, L., Machado, M., et al. (2005). Management of Hepatic Hemangiomas: A 14-Year Experience. J. Gastrointest. Surg. 9, 853-859. doi:10.1016/j.gassur.2005.01.292

Ji, J., Gao, J., Zhao, L., Tu, J., Song, J., and Sun, W. (2016). Computed TomographyGuided Radiofrequency Ablation Following Transcatheter Arterial Embolization in Treatment of Large Hepatic Hemangiomas. Medicine (Baltimore) 95, e3402. doi:10.1097/MD.0000000000003402

Ketchum, W. A., Lin-Hurtubise, K. M., Ochmanek, E., Ishihara, K., and Rice, R. D. (2019). Management of Symptomatic Hepatic "Mega" Hemangioma. Hawaii J. Med. Public Health 78, 128-131. doi:10.1097/meg.0000000000001413

Li, G. W., Chen, Q. L., Jiang, J. T., and Zhao, Z. R. (2003). The Origin of Blood Supply for Cavernous Hemangioma of the Liver. Hepatobiliary Pancreat. Dis. Int. 2, 367-370. doi:10.3748/wjg.v3.i3.147

Li, Y., Jia, Y., Li, S., Wang, W., Wang, Z., Wang, Y., et al. (2015). Transarterial Chemoembolization of Giant Liver Haemangioma: A Multi-center Study with 836 Cases. Cell Biochem Biophys 73, 469-472. PMID: 27352340. doi:10.1007/ s12013-015-0680-y
Liu, F., Yu, X., Liang, P., Cheng, Z., Han, Z., and Yu, J. (2018). Ultrasonographyguided Percutaneous Microwave Ablation for Large Hepatic Cavernous Haemangiomas. Int. J. Hyperthermia 34, 1061-1066. doi:10.1080/ 02656736.2017.1392045

Miura, J. T., Amini, A., Schmocker, R., Nichols, S., Sukato, D., Winslow, E. R., et al. (2014). Surgical Management of Hepatic Hemangiomas: a Multi-Institutional Experience. $H P B$ 16, 924-928. doi:10.1111/hpb.12291

Özden, İ., Poyanlı, A., Önal, Y., Demir, A. A., Hoş, G., and Acunaş, B. (2017). Superselective Transarterial Chemoembolization as an Alternative to Surgery in Symptomatic/Enlarging Liver Hemangiomas. World J. Surg. 41, 2796-2803. doi:10.1007/s00268-017-4069-5

Ramachandran, V., Garg, R., Pal, S., Gamanagatti, S., Srivastava, D., and Jain, V. (2010). Spontaneous Rupture of a Giant Hepatic Hemangioma Sequential Management with Transcatheter Arterial Embolization and Resection. Saudi J. Gastroenterol. 16, 116-119. doi:10.4103/13193767.61240

Schnelldorfer, T., Ware, A. L., Smoot, R., Schleck, C. D., Harmsen, W. S., and Nagorney, D. M. (2010). Management of Giant Hemangioma of the Liver: Resection versus Observation. J. Am. Coll. Surgeons 211, 724-730. doi:10.1016/ j.jamcollsurg.2010.08.006

Srivastava, D. N., Gandhi, D., Seith, A., Pande, G. K., and Sahni, P. (2001). Transcatheter Arterial Embolization in the Treatment of Symptomatic Cavernous Hemangiomas of the Liver: a Prospective Study. Abdom. Imaging 26, 510-514. doi:10.1007/s00261-001-0007-x

Szejnfeld, D., Nunes, T. F., Fornazari, V. A. V., Matos, C. A. L. d., Gonzalez, A. M., D'Ippolito, G., et al. (2015). Transcatheter Arterial Embolization for Unresectable Symptomatic Giant Hepatic Hemangiomas: Single-center Experience Using a Lipiodol-Ethanol Mixture. Radiol. Bras 48, 154-157. doi:10.1590/0100-3984.2014.0063

Torkian, P., Li, J., Kaufman, J. A., and Jahangiri, Y. (2021). Effectiveness of Transarterial Embolization in Treatment of Symptomatic Hepatic Hemangiomas: Systematic Review and Meta-Analysis. Cardiovasc. Intervent Radiol. 44, 80-91. doi:10.1007/s00270-020-02611-5

Toro, A., Mahfouz, A.-E., Ardiri, A., Malaguarnera, M., Malaguarnera, G., Loria, F., et al. (2014). What Is Changing in Indications and Treatment of Hepatic Hemangiomas. A Review. Ann. Hepatol. 13, 327-339. doi:10.1016/s16652681(19)30839-7

Xue, T., Feng, W., Yu, H., Zhu, M., Fei, M., Bao, Y., et al. (2017). MetastasisAssociated Protein 1 Is Involved in Angiogenesis after Transarterial Chemoembolization Treatment. Biomed. Res. Int. 2017, 1-10. doi:10.1155/ 2017/6757898

Yoon, S. S., Charny, C. K., Fong, Y., Jarnagin, W. R., Schwartz, L. H., Blumgart, L. H., et al. (2003). Diagnosis, Management, and Outcomes of 115 Patients with Hepatic Hemangioma. J. Am. Coll. Surgeons 197, 392-402. doi:10.1016/S10727515(03)00420-4

Conflict of Interest: The authors declare that the research was conducted in the absence of any commercial or financial relationships that could be construed as a potential conflict of interest.

Publisher's Note: All claims expressed in this article are solely those of the authors and do not necessarily represent those of their affiliated organizations, or those of the publisher, the editors, and the reviewers. Any product that may be evaluated in this article, or claim that may be made by its manufacturer, is not guaranteed or endorsed by the publisher.

Copyright (C) $2021 \mathrm{Liu}, \mathrm{You}, \mathrm{Zhu}, \mathrm{Li}$, Wu, Wang, Wang, Zhu, Gu, Peng and Zheng. This is an open-access article distributed under the terms of the Creative Commons Attribution License (CC BY). The use, distribution or reproduction in other forums is permitted, provided the original author(s) and the copyright owner(s) are credited and that the original publication in this journal is cited, in accordance with accepted academic practice. No use, distribution or reproduction is permitted which does not comply with these terms. 